\title{
Prácticas de lectura en primer año de la Universidad. Un estudio sobre las representaciones sociales de la lectura en alumnos ingresantes a la UNPA
}

\section{Reading practices in the first year of University. A study of social representations in first- year students of UNPA}

\author{
Andrea Carolina González, Mónica Musci \\ profeandreag@gmail.com,mmusci@uarg.unpa.edu.ar \\ UNPA-ICIC-UARG \\ Río Gallegos - Santa Cruz - Argentina
}

Recibido: 05/04/2020. Aceptado: 11/09/2020

\section{RESUMEN}

El estudio de las representaciones sociales, concepto que proviene de la Psicología Social, ha cobrado cierta relevancia en el campo de la educación en el último tiempo debido a que permite develar esquemas de percepción y pensamiento socialmente compartidos por un grupo sobre aspectos de la realidad (Jodelet, 1986). Así, las representaciones sobre la lectura y los significados que tienen sobre ella los alumnos en el ámbito universitario influyen en la manera como se efectúa esta actividad, pudiendo constituir un obstáculo o una ayuda para su desempeño académico. Siguiendo este enfoque, la presente comunicación constituye el informe final realizado en el marco de un proyecto de investigación del área de lingüística que analiza las representaciones sociales sobre la lectura (RSL) en los alumnos ingresantes de la UNPA-UARG. Particularmente, nos ocupamos aquí de presentar algunos resultados de una encuesta realizada a estudiantes ingresantes de distintas carreras. La indagación puso énfasis en rastrear dos aspectos presentes en las representaciones sociales: la dimensión informativa y la dimensión actitudinal. La finalidad es analizar la incidencia de las RSL en sus prácticas de lectura académica y construir un perfil del sujeto lector que comprenda diversos aspectos como las historias escolares previas, las motivaciones, los hábitos de lectura y la percepción de la lectura en relación con el aprendizaje.

Palabras Clave: Lectura académica; representaciones sociales; encuesta; perfil lector de los ingresantes.

\begin{abstract}
The study of social representations, a concept that comes from Social Psychology has gained some relevance in the field of education in recent times because it allows us to reveal socially shared perception and thinking schemes by a group about reality aspects (Jodelet, 1986). Thus, the representations about reading and the meanings that students have about it in the university environment have influence in the way in which activity is carried out, being able to constitute an obstacle or an aid for their academic performance. Following this approach, this communication constitutes the final report made within the framework of a research project in the area of linguistics that analyzes social representations about reading (RSR) in first-year students of UNPA-UARG. Mainly, we present here some results of a survey of incoming students from different UNPA university careers. The inquiry put emphasis on tracking two aspects present in social representations: the informative dimension and the attitudinal dimension. The purpose is to analyze the incidence of RSR in their academic reading practices and build a profile of the reading subject that includes various aspects such
\end{abstract}


as previous school stories, motivations, reading habits and the perception of reading in relation to learning.

Keywords: Academic reading; social representations; survey; reading profile of first-year students.

\section{INTRODUCCIÓN}

La lectura es una práctica esencial en la vida del alumno universitario, a partir de ella se transmiten y se construyen nuevos conocimientos, además, su dominio determinará la incorporación de los nuevos miembros a la comunidad académica y profesional. Pero, ¿cómo leen los alumnos que ingresan a la universidad? ¿Qué factores inciden en la forma de leer de los nuevos estudiantes? Sin duda estas son cuestiones que atraviesan todas las áreas disciplinares, sin embargo, como lo señala Carlino (2011), muchas veces esta práctica pasa inadvertida. Se presupone que un alumno universitario ya debe tener desarrollada esta habilidad, no se tiene en cuenta la manera real en que los estudiantes perciben y practican la lectura, ni constituye un objeto de enseñanza en el nivel superior.

En relación con esta problemática se han realizado diferentes estudios en instituciones de nivel superior en Argentina que plantean la dificultad del acceso a la lectura comprensiva de los textos académicos en los ingresantes. Algunos de ellos indican que, en gran medida, esta situación se produce debido a un desfasaje entre las representaciones sociales que tienen los alumnos acerca de las prácticas de lectura y aquellas que sostiene la comunidad discursiva académica-universitaria (di Stéfano y Pereira, 1997, 2004; Sosa de Montyn, 2006).

El presente trabajo sobre las representaciones sociales de la lectura coincide y acoge los planteamientos teóricos señalados anteriormente y que explicitamos a continuación: consideramos que las representaciones sociales de la lectura se construyen a partir de las experiencias previas de cada individuo, los valores y los discursos dominantes que circulan sobre ella; estos "saberes" generan actitudes y orientan la forma en que los alumnos llevan a cabo esta práctica en la universidad. En relación con lo expuesto Abric destaca la manera en que la representación social orienta las acciones de las personas: "funciona como un sistema de interpretación de la realidad que rige las relaciones de los individuos con su entorno físico y social, ya que determina sus comportamientos o sus prácticas. Es una guía para la acción" (2001:13). A su vez, el valor de estudiar las representaciones sociales de la lectura en los estudiantes ingresantes reside en su utilidad didáctica, ya que pueden constituir una ayuda o un obstáculo para el aprendizaje y son modificables.

En el marco de un proyecto de investigación más amplio ${ }^{2}$ que se dedica al estudio de las intervenciones glotopolíticas $^{3}$ en la universidad, nos centraremos aquí en describir las representaciones sociales sobre la lectura que poseen los alumnos ingresantes de la UNPAUARG y analizar su incidencia en la selección de operaciones cognitivas para resolver tareas de interpretación. Para ello, explicaremos algunas conceptualizaciones sobre nuestro objeto de estudio y expondremos los resultados de una encuesta que indagó sobre las dimensiones informativa y actitudinal de las representaciones sociales sobre la lectura (RSL). Nuestro

\footnotetext{
1 Jodelet (1986) considera las RS como el conjunto de “conocimientos de sentido común” que permiten la comprensión de la realidad.

${ }^{2}$ PI: 29/A432 "Regulaciones discursivas: intervenciones glotopolíticas en la Universidad"

3 Como lo explica Arnoux (2000), el término de Glotopolítica designa las diversas formas en que una sociedad actúa sobre el lenguaje, tanto de manera explícita como implícita; en este sentido la lectura académica se inserta en este campo pues nos referimos a un modo específico de esta práctica que es aprobado, requerido y regulado por la comunidad discursiva universitaria.
} 
objetivo es obtener datos que contribuyan a explicar los obstáculos que podrían estar dificultando sus prácticas lectoras universitarias.

La encuesta fue realizada en una cohorte de estudiantes ingresantes de distintas carreras que cursaron la materia Análisis del Discurso durante el segundo cuatrimestre del año 2019. La indagación apuntó a recabar datos acerca de qué, cuánto y cómo leen, en qué soportes, cómo acceden a sus materiales de lectura, qué características tuvieron sus experiencias de lectura en la escuela media y qué cambios en su forma de leer ha implicado el ingreso a la universidad. El análisis cualitativo y cuantitativo de los datos obtenidos nos permite caracterizar a los alumnos ingresantes como lectores medios, es decir, no son ajenos a la cultura escrita, pero tampoco se perciben a sí mismos como lectores de textos académicos que puedan autorregular sus propios procesos de comprensión.

El trabajo se estructura de la siguiente manera: en primer lugar vamos a describir nuestro objeto de estudio (RSL) definiendo conceptos referidos a la lectura en general, la lectura académica en particular y la importancia de las representaciones sociales para su caracterización. En segundo lugar haremos una reseña de trabajos científicos previos que describen al lector universitario. En tercer lugar precisaremos algunos aspectos del instrumento de análisis utilizado y presentaremos los resultados más significativos de la encuesta, a fin de construir un perfil lector de los estudiantes ingresantes de la UNPA-UARG. Para esbozar este perfil focalizaremos la atención en los siguientes ejes: la relación que manifiestan los estudiantes con la lectura, sus experiencias lectoras-escolares previas y las representaciones, propósitos y obstáculos que presenta la lectura en la universidad. Finalmente, expondremos las principales conclusiones de este trabajo.

\section{MARCO TEÓRICO}

\subsection{Lectura y representaciones sociales}

¿Qué es leer? Esta pregunta plantea un concepto que ha sido objeto de intensa reflexión y puede tener diferentes acepciones dependiendo del enfoque desde el que se estudie. Particularmente, esta investigación parte de una perspectiva psicosociocognitiva que entiende la lectura como una práctica social que presenta dos aspectos (Bourdieu, 1988): un componente material (cuerpos/lectores, objetos/libros y espacios involucrados en la actividad/bibliotecas, universidades) y otro cognitivo-social, constituido por las representaciones compartidas por un grupo acerca del valor, el sentido y el modo correcto de llevarlas adelante.

La lectura tiene un papel central en la socialización del sujeto; su carácter de actividad regular y repetida siempre de manera análoga provoca que los individuos la internalicen a través de esquemas de percepción, valoración y clasificación. Como efecto de esta exposición repetida a las prácticas, los sujetos se integran a una sociedad determinada. Por esta razón, las prácticas sociales pueden historizarse y están sujetas a cambios diacrónicos. El estudio de las representaciones sociales, incluye entonces la necesidad de incorporar esta dimensión imaginaria compartida.

El concepto de Representación social proviene de la Psicología Social inspirada en Moscovici (1989). Esta noción vincula la visión de un objeto con la pertenencia sociocultural del sujeto. Es un conocimiento del sentido común, espontáneo, proveniente de saberes heredados de la tradición, de la educación y la comunicación social, que modela la mirada que el individuo tiene del mundo y sus interacciones sociales. Las representaciones sociales son "formas de 
conocimiento socialmente elaboradas y compartidas que tienen una meta práctica y cooperan en la construcción de una realidad común a un conjunto social" (Jodelet, 1989:36).

Esta autora considera a las representaciones fenómenos complejo, compuestos por elementos diversos: informativos, cognitivos, ideológicos, normativos, creencias, valores, actitudes, opiniones, imágenes, organizados como un saber sobre la realidad. Se configuran en la intersección de sistemas de pensamientos más amplios ideológicos y culturales, influidos por el estado de conocimientos científicos y también por la condición social y las experiencias privadas y afectivas de los individuos. El concepto de representación social ha ido cobrando cada vez más importancia en el campo de las Ciencias Sociales, lo que produjo una gran cantidad de investigaciones cuyo propósito fue describirlas en relación con objetos y prácticas diversos.

Para la investigación en lectura y escritura en la educación superior, resulta particularmente útil desde el punto de vista teórico algunas de las características de las representaciones: su cualidad anticipatoria, ya que preceden y orientan la acción de los sujetos; su carácter dinámico y en especial, su maleabilidad, es decir la posibilidad de transformación de la que son susceptibles. En especial, esta última puede ser una clave de acceso para diseñar dispositivos didácticos encaminados a ajustar las prácticas de lectura de los ingresantes a las convenciones y expectativas corrientes en el ámbito de la Universidad.

\subsection{Leer en la universidad}

Nuestra concepción de la lectura como práctica social nos conduce a considerar que está históricamente situada, es decir, que el contexto en el que se inscribe la práctica incide en el modo en que se la lleva a cabo. Por lo tanto, lo primero que advertimos es que la lectura es una práctica inherente a la vida universitaria donde -por lo general- no se cuestiona si el alumno es un lector, sino que se asume que debería serlo. En segundo lugar, creemos que existen regulaciones, en su mayoría implícitas, que orientan al alumno hacia los modos específicos que requiere la práctica de lectura en este ámbito.

Una formación de grado universitario exige, además de la formación disciplinar, el desarrollo de determinadas competencias de lectura y escritura propias de esa comunidad discursiva. En este sentido, señala Sosa de Montyn (2017:20): "Una institución como la universidad demanda la lectura de textos vinculados con el quehacer científico de un alto grado de complejidad. La interpretación de los mismos exige al estudiante el desarrollo de habilidades de lectura y escritura específicas a fin de perfeccionar su competencia discursiva".

Asimismo, Nogueira y Robbio (2010: 22) destacan algunas características las comunidades discursivas académicas, como:

- La producción de conocimientos controlados por instancias institucionales de evaluación y control;

- Un repertorio específico de géneros discursivos complejos (monografía, tesis, tesina, ponencia, artículo de investigación, proyecto de investigación, informe de lectura o de investigación, solicitud de beca, dictamen de concurso docente, lección inaugural, conferencia, entre otros);

- El estatuto jerarquizado de sus miembros (estatuto que determina quién puede producir qué géneros y para quién $[\ldots])$;

Por lo expuesto, queda de manifiesto que la comunidad discursiva académica requiere de lectores que asuman un rol activo frente a los textos -principalmente científicos y académicos- y donde la práctica de la lectura se considera fundamentalmente una herramienta 
para generar conocimientos y favorecer el desarrollo de competencias que garanticen el desarrollo profesional (Fernández y Ojeda, 2018).

Cuando se lee para aprender - lo cual es la principal finalidad de la lectura en la Universidad-, el lector efectúa una serie de procesos cognitivos tales como leer, representar, evocar, conectar la información nueva con los conocimientos previos, reestructurar la información, evaluar la comprensión; estas acciones le permiten interiorizar por más tiempo el contenido que se quiere aprender (Parodi, 2010).Este tipo de lectura que involucra al aprendizaje es la que se conoce como epistémica y exige un compromiso cognitivo por parte de los sujetos: posicionarse frente al texto, formular juicios y justificaciones argumentativas (Boatto et al, 2016). Por otra parte, respecto a las estrategias que se activan cuando se realiza una lectura epistémica Solé (2000:83) plantea:

\begin{abstract}
El lector se ve inmerso en un proceso que lo lleva a autointerrogarse sobre lo que lee, a establecer relaciones con lo que ya sabe, a revisar los términos que le resultan nuevos, a efectuar recapitulaciones y síntesis frecuentes, a subrayar, a tomar notas [...] Cuando se lee para estudiar, es habitual y es de gran ayuda elaborar resúmenes y esquemas sobre lo leído, anotar lo que constituye una duda, volver a leer el texto u otros que puedan contribuir en el aprendizaje, etc. Cuando leemos para aprender, las estrategias responsables de una lectura eficaz y controlada se actualizan de forma integrada y consciente permitiendo la elaboración de significados que caracterizan al aprendizaje.
\end{abstract}

En relación con lo expuesto podemos afirmar que la Universidad demanda que los alumnos se posicionen como lectores que autorregulen su propio proceso de lectura; sin embargo, la forma en que los estudiantes ingresantes leen deriva de sus características peculiares y de sus historias escolares previas que constituyen una parte de sus representaciones sociales sobre la lectura.

\title{
2.3 Leer en el siglo XXI
}

Al hablar de lectura académica no podemos dejar de remitirnos al contexto histórico y social en que viven y se forman los estudiantes hoy: la era digital. Esta época ha producido profundos cambios en las prácticas de la lectura a partir de la incorporación de las nuevas tecnologías de la comunicación. El simple acto de trasladar la lectura del texto impreso al digital -tarea cada vez más usual en el ámbito académico- conlleva la modificación de una práctica que durante siglos se había mantenido inalterable. En relación con esto, encuestas realizadas en la UNPA (Musci, 2015) revelan un avance significativo de lo que se conoce como lectura electrónica, lectura digital o e-Reading.

Por otra parte, Gutiérrez (2009), al referirse a este fenómeno, cita la investigación de Julie Coiro (2003) acerca las nuevas competencias en el perfil del ciber-lector, destacando alguna de ellas:

capacidades para buscar, ubicar y establecer conexiones entre recursos desde múltiples y diversas perspectivas; la habilidad para manipular innumerables bases de datos y para la utilización de múltiples motores de búsqueda; la capacidad de asociación y de navegación para buscar, recorrer, encontrar, seleccionar, añadir, eliminar, fraccionar, reordenar y extraer de los textos en línea, con el menor esfuerzo posible, la información que necesitan o que les interesa e incluso, la facultad de realizar descubrimientos, por .accidente., de documentos electrónicos con información que inicialmente no se buscaba, pero que termina por ser de gran utilidad [serendipia]

En este sentido, observamos que las nuevas tecnologías y soportes asociadas con Internet hacen más accesible los textos a un gran número de estudiantes, posibilitando su acceso casi inmediato a través de las pantallas de sus propios dispositivos electrónicos (celular, notebook, tablet, etc.); sin embargo, la lectura en el entorno digital también lleva a la tarea de desarrollar 
nuevas habilidades en el proceso de comprensión que deben ser atendidas desde todos los niveles de la educación.

\subsection{Antecedentes}

Los procesos involucrados en la lectura han sido analizados exhaustivamente por la Psicología Cognitiva (Goodman, 1982; Parodi, 2011) y han producido una serie de modelos centrados en las operaciones que un sujeto lleva a cabo en interacción con el texto. Sin embargo, pocos se han ocupado de la regulación selectiva o automática de las operaciones cognitivas involucradas en la lectura de textos complejos. ¿Qué impulsa a un lector a tomar determinadas actitudes frente a un texto, a buscar información a partir de la lectura, a confrontar posturas diversas a partir de la lectura de distintas fuentes? Estas y otras preguntas se plantean las investigadoras Mariana di Stéfano y Cecilia Pereira (1997) al establecer como objetivos de su investigación la necesidad de contemplar la incidencia de las representaciones sociales sobre la lectura y la escritura de los alumnos con el fin de intervenir en ellas para favorecer positivamente que los estudiantes respondan a las exigencias de la vida académica. Estas autoras señalan la necesidad de poner en correlación las representaciones que tienen los lectores sobre lo que es leer, la situación comunicativa, su rol como lector y la finalidad de la lectura.

Estas representaciones no son individuales sino compartidas, en este caso, por los grupos de ingresantes a la universidad, producto en parte de sus experiencias como lectores en trayectos educativos anteriores. La articulación de la teoría de las representaciones sociales y las conceptualizaciones sobre los procesos de lectura ha conducido a conectar las investigaciones sobre las prácticas lingüísticas con los estudios tradicionales en Sociología encaminados a obtener información sobre prácticas sociales a partir de las encuestas sobre la lectura como fuente de información (Lahire, 2004).

En América Latina y en particular en Argentina se han llevado a cabo diversas encuestas, la más conocida es la Encuesta Nacional de Consumos Culturales, para medir, entre otras cosas, los hábitos lectores de la población en términos de libros leídos, lectura de periódicos, historietas, lectura digital, uso de bibliotecas, actitud y valoración de la práctica. También se han llevado a cabo diferentes encuestas en las instituciones de nivel superior encaminadas a obtener información sobre hábitos, actitudes ante la práctica, valoración y propósitos que los alumnos, en especial los ingresantes, ponen en juego al realizar actividades de lectura (Sosa de Montyn et al, 2006; Musci, 2015). El equipo de la cátedra de Semiología publicó los resultados de una encuesta, administrada en la UBA y elaborada a partir de los aportes de la Sociología de la lectura, que buscó obtener datos para evaluar la incidencia de las lecturas históricas y actuales en el desempeño de estudiantes del nivel superior (di Stéfano y Pereira, 2014). El análisis cuantitativo y cualitativo realizado permite caracterizar al estudiante como un lector "medio básico", con una representación de pertenencia a la cultura escrita, que privilegia la lectura literaria y periodística y que representa la lectura como práctica emotiva e identitaria. Estas representaciones son atribuidas a una matriz escolar que, al reiterar los mismos modos de aproximación a la lectura, no ha invitado al alumno a familiarizarse con géneros y experiencias de lectura variadas.

\section{MATERIALES Y MÉTODOS}

3.1 Descripción de la muestra 
La población seleccionada para esta investigación está conformada por alumnos ingresantes de ambos sexos de la Unidad académica Río Gallegos que cursan la materia Análisis del Discurso, una materia común a todas las carreras de la UNPA en primer año, de carácter obligatorio y de dictado anual. La administración de la encuesta estuvo a cargo de la becaria y se realizó durante el segundo cuatrimestre del año 2019. En total se encuestaron 44 alumnos correspondientes a las carreras: Comunicación Social, Tecnicatura en Acompañamiento Terapéutico, Ingeniería Química, Licenciatura en Sistemas e Ingeniería en Recursos renovables. La edad de los encuestados oscila entre los 18 y los 60 años de edad y el $90 \%$ de ellos son ingresantes, el resto son recursantes de la materia que se encuentran en 2 do año de sus respectivas carreras.

\subsection{Descripción de la encuesta}

El instrumento utilizado para recolectar información sobre las representaciones sociales sobre la lectura en ingresantes a la UNPA fue una encuesta semiestructurada, diseñada en base a instrumentos similares aplicados en investigaciones relacionadas con nuestro tema y objetivo $^{4}$. En un primer momento se desarrolló una prueba piloto con un grupo de alumnos, lo cual permitió evaluar el cuestionario, validar la estrategia de recolección de información y puntualizar las categorías de análisis. Luego, se procedió a reformular preguntas tanto abiertas como cerradas orientadas a rastrear dos aspectos presentes en las representaciones sociales: la dimensión informativa y la dimensión actitudinal.

La primera, se refiere a una forma particular de conocimiento, ideas dispersas provenientes de situaciones de acercamiento con el objeto de estudio, en este caso, la lectura. Estas representaciones sociales se generan desde varias fuentes: los medios de comunicación, la interacción con los grupos sociales y la educación. Esta dimensión responde a la interrogación: ¿qué se sabe sobre el objeto social en cuestión? (Araya, 2002). Las preguntas de la encuesta que la abordan son las que refieren a las experiencias de lectura previa de los ingresantes y -también- las que representan una valoración de la lectura en el ámbito académico universitario.

La segunda dimensión toma en cuenta la actitud, que tiene una relación directa con la forma de actuar del sujeto frente a una situación concreta, orienta su conducta, su discurso y su accionar emocional (Araya, 2002). Para observar esta dimensión, se solicitó a los ingresantes que describieran sus obstáculos de lectura más frecuentes y las formas de abordarlos, es decir, se les pidió que elijan una forma de resolver las dificultades de lectura que planteaban.

La encuesta original ${ }^{5}$ consta de 12 preguntas, 9 con opción múltiple y 4 preguntas abiertas, que permiten al encuestado responder con su propio vocabulario de forma más independiente. El cuestionario aborda distintos asuntos como: motivación, dedicación, propósitos de lectura, tipos de texto y soportes utilizados. Si bien todas son útiles para dibujar un perfil lector de los alumnos ingresantes, en este trabajo sólo nos centramos en 6 cuestiones, aquellas que dan cuenta de las representaciones sociales sobre la lectura en las dimensiones anteriormente descriptas.

Los cuadros que siguen muestran las preguntas seleccionadas y los ejes en que las agrupamos para su análisis:

\footnotetext{
${ }^{4}$ Trabajamos fundamentalmente con los aportes de la Sociología de la lectura que privilegia el empleo de la encuesta como instrumento de recolección de datos.

${ }^{5}$ En la sección Anexos se adjunta un modelo completo de la encuesta realizada.
} 
Tabla 1: Eje relación con la lectura

\begin{tabular}{|c|l|l|}
\hline VARIABLE & \multicolumn{1}{|c|}{ PREGUNTA } & \multicolumn{1}{|c|}{ CATEGORÍA DE RESPUESTA } \\
\hline Gusto & $\begin{array}{l}\text { ¿Cuál de las siguientes } \\
\text { frases explica mejor tu } \\
\text { relación con la lectura? }\end{array}$ & $\begin{array}{l}\text { a) No puedo vivir sin la lectura } \\
\text { b) Me gusta mucho leer } \\
\text { c) Me gusta leer de vez en cuando } \\
\text { d) Me gusta poco leer } \\
\text { e) No me gusta nada leer }\end{array}$ \\
\hline Motivación & $\begin{array}{l}\text { 2. ¿Cuáles son las razones } \\
\text { principales que te llevan a leer? }\end{array}$ & $\begin{array}{l}\text { a) Informarme sobre algún tema de } \\
\text { interés } \\
\text { b) El deseo de conocer cosas nuevas } \\
\text { c) Cumplir con las actividades } \\
\text { académicas } \\
\text { d) Deseo expresarme mejor } \\
\text { e) El placer que encuentro en la lectura } \\
\text { f) Crecimiento personal } \\
\text { g) Para ser un mejor profesional } \\
\text { h) Para huir de las preocupaciones } \\
\text { i) Para ser culto } \\
\text { j) Otra, ¿cuál? }\end{array}$ \\
\hline
\end{tabular}

Tabla 2: Eje experiencias escolares previas

Comparación
entre la lectura
en la escuela
media y en la
universidad

3. Pensá en la prácticas de lectura que realizabas en la escuela secundaria, ¿qué diferencias encontrás con las que realizás ahora en la universidad?

Abierta

Tabla 3: Eje la lectura en la universidad

\begin{tabular}{|c|c|c|}
\hline $\begin{array}{l}\text { Representación } \\
\text { de la lectura en } \\
\text { la universidad }\end{array}$ & $\begin{array}{l}\text { 4. ¿Por qué es importante leer } \\
\text { bien en la universidad? }\end{array}$ & Abierta \\
\hline Dificultades & $\begin{array}{l}\text { 5. ¿Cuáles creés que son los } \\
\text { obstáculos de lectura más } \\
\text { frecuentes en la universidad? } \\
\text { ¿Podés dar un ejemplo? }\end{array}$ & Abierta \\
\hline Actitud & $\begin{array}{l}\text { 6. ¿Qué hacés ante las } \\
\text { dificultades de lectura que se te } \\
\text { presentan? }\end{array}$ & $\begin{array}{l}\text { a) Dejás de leer } \\
\text { b) Completás la lectura aunque no } \\
\text { entiendas el texto } \\
\text { c) Lo releés } \\
\text { d) Buscás información } \\
\text { complementaria en Internet } \\
\text { e) Buscás las palabras desconocidas } \\
\text { f) Lo relacionás con otras lecturas } \\
\text { g) Consultás con el profesor } \\
\text { h) Consultás con un compañero } \\
\text { i) Otras estrategias: }\end{array}$ \\
\hline
\end{tabular}




\section{RESULTADOS, ANÁLISIS Y DISCUSIÓN}

A continuación se presenta una interpretación cualitativa de los resultados organizados en torno a tres ejes temáticos:

I. La relación de los alumnos con la lectura: preguntas 1 y 2

II. Sus experiencias escolares previas: pregunta 3

III. La lectura en el nivel superior: preguntas 4,5 y 6 .

Estos resultados son puestos en correlación con estudios del mismo tipo que se llevaron a cabo en otras Universidades, y constituyen otra contribución para una descripción más ajustada de las prácticas de lectura en la educación superior y de las representaciones que tienen los actores involucrados acerca de ellas.

I. La relación de los alumnos con la lectura:

En cuanto a la relación de los estudiantes con la lectura se realizaron dos preguntas con varias opciones relacionadas con su imagen como lectores (¿cuál de las siguientes frases explica mejor tu relación con la lectura?) y los motivos principales que los llevan a leer (¿cuáles son las razones principales que te llevan a leer?). La sistematización de las preguntas ha arrojado los siguientes resultados:

Gráfico 1

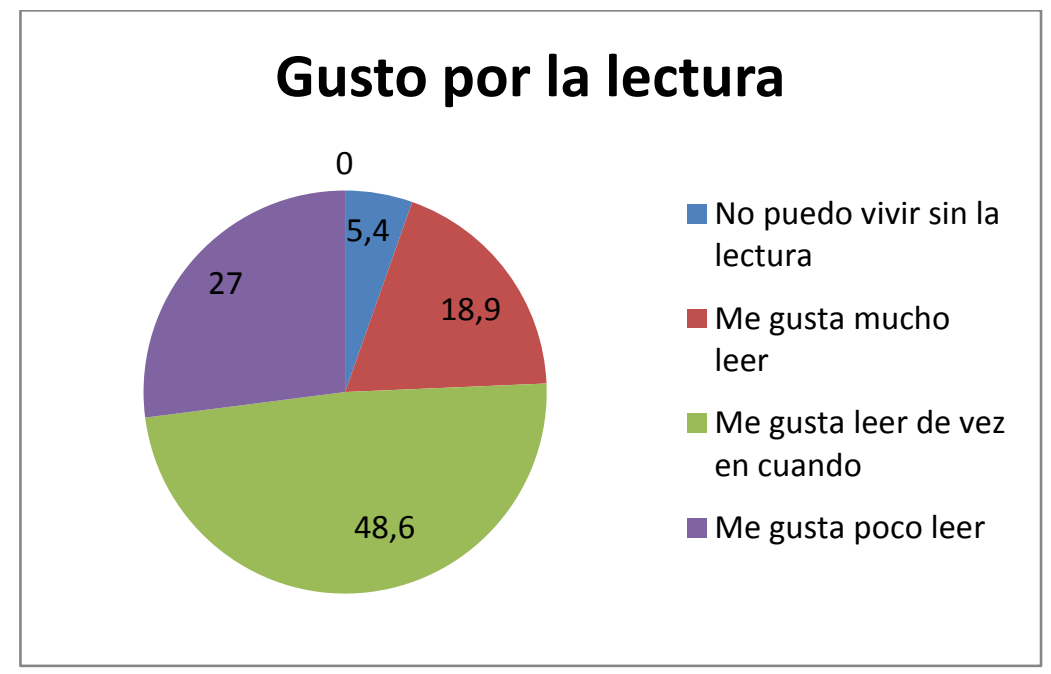

El estudio de la primera cuestión revela que a la mayoría de los encuestados se ven a sí mismos como lectores ocasionales, es decir, que sólo les gusta leer de vez en cuando $(48,6 \%)$, incluso un $27 \%$ refiere que le gusta poco leer. Estos porcentaje representan un desafío importante e inquietante ya que se trata de alumnos que ingresan a la Universidad, ámbito en el cual la lectura será una de las prácticas más importantes (si no la más importante) para la adquisición acumulación de conocimientos. El número de quienes declaran gusto e incluso pasión por la lectura (18,9 \% y 5,4 \% respectivamente) es reducido en relación con la suma de quienes leen de vez en cuando y a quienes les gusta poco leer y plantea un verdadero desafío para la educación superior. Aquí nos planteamos un nuevo interrogante: ese de vez en cuando, ¿incluye la lectura académica? ¿O cuando responden por el gusto de leer están pensando en otras lecturas (literarias, de entretenimiento) y no en el estudio? Este resultado puede valorarse en relación con los siguientes. 


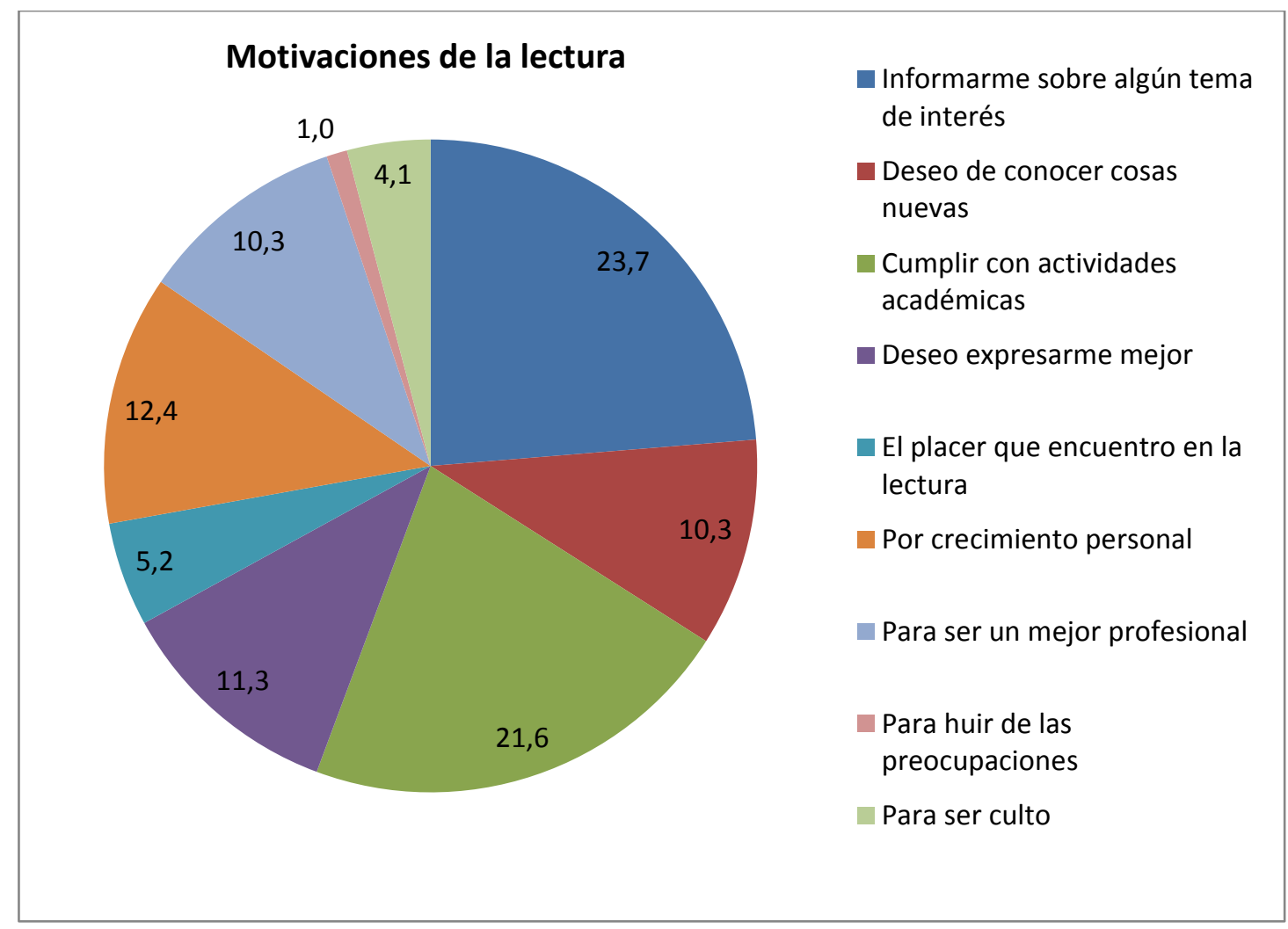

Respecto a la segunda cuestión observamos que las principales motivaciones de lectura tienen que ver con informarse sobre algún tema de interés $(23,7 \%)$ y cumplir con las actividades académicas $(21,6 \%)$. Podemos considerarlas dos modos de acercamiento a la lectura, la primera como una práctica impulsada por la curiosidad, más libre y desestructurada; la segunda, motorizada por un proyecto de vida personal que somete su desarrollo a la sistematicidad del sistema educativo. Los demás motivos que aparecen pueden asociarse a estos dos principales: por un lado, el objetivo de ser un mejor profesional está asociado con el cumplimiento de las actividades académicas; por el otro, el deseo de conocer cosas nuevas, el placer, el crecimiento personal, el ser culto, están más cerca del 'interés' y la necesidad de estar informado. Encontramos en estas formulaciones rasgos de los dos modelos predominantes de lectura que subsisten en los últimos años del siglo XX y son producto de procesos históricos que se remontan al siglo anterior ya descriptos por Anne-Marie Chartier (2002) en Francia: por un lado, el modelo de la lectura formativa (académica en este caso) gesto colectivo y obligatorio de capas generacionales cuya instrucción es un deber del Estado, que selecciona (y controla) las lecturas y recela de los malos libros, la pseudoliteratura y la falsa ciencia. Por otro lado, el modelo de consumo, que tiene su origen fuera de la escuela y que nace al impulso de la cultura de masas y de la difusión masiva de los textos impresos; valora la lectura de actualidad que exige un constante reaprovisionamiento de los escritos (periódicos, revistas, las últimas novelas) y considera al lector un ciudadano libre que lee en soledad y según su voluntad. Chartier señala que ambos se fusionan en la escuela contemporánea, que los asume sin contradicciones, y su articulación se sostiene en el consenso social que formula que 'Leer es un gesto incondicionalmente positivo'. Esta percepción de los alumnos está fuertemente arraigada, todas las respuestas valoran la práctica de la lectura positivamente, aún cuando ellos mismos no la practiquen asiduamente. 
Estos resultados ubican a los encuestados como lectores medios a los cuales la cultura escrita no les es totalmente ajena (di Stéfano y Pereira, 2014). Además, lo datos expuestos pueden ser entendidos en relación con los fines de la lectura en la universidad, es decir, los estudiantes dedican la mayor parte de su tiempo a leer textos de estudio que les generan mayores dificultades que los literarios, como podemos observar en los resultados de la siguiente pregunta que analizamos.

\section{Las experiencias escolares previas}

Respecto a los resultados de las encuestas asociados a sus experiencias previas de lectura, se les solicitó que señalaran diferencias entre las formas de leer en la escuela secundaria con las de la universidad. En este punto la mayor parte de los encuestados revelan que existen notables diferencias entre las prácticas de lectura que realizaban en el nivel medio y las que realizan hoy en el nivel superior. Sus experiencias previas de lectura están relacionadas principalmente con la literatura y con textos didácticos a los que se les aplicaban preguntas dirigidas a buscar una respuesta que se encontraba en el mismo texto; tampoco manejaban variedad de fuentes. Por otro lado, la lectura académica les supone nuevos desafíos relacionados principalmente con la complejidad y formas de leer que les resultan desconocidas. Se citan a continuación algunas respuestas que ilustran estos resultados:

"En la secundaría leíamos por obligación, en la universidad tiene que ver más con la carrera que a uno le gusta."

"Los textos en el secundario te decían la respuesta, en la universidad son más complejos y extensos."

“En la secundaria los textos eran más sencillos, no había la misma exigencia."

"En la universidad leer es una responsabilidad de cada uno, en la secundaria era más relajado."

"La lectura universitaria requiere mayor atención, hay más cantidad de textos, muchos autores, los textos presentan un lenguaje más complejo."

"En la secundaria sólo leía cuando había evaluaciones, en la universidad estás leyendo todo el tiempo"

"En la secundaria sólo leíamos en lengua."

Estos resultados son coincidentes con los obtenidos por Pereira y Di Stéfano (2014) que arrojan que un gran porcentaje de alumnos señalan que en la Universidad leen más y mejor (más tiempo, libros completos, con mejores estrategias). Por otro lado, es interesante constatar la conciencia que tienen los alumnos de las nuevas exigencias de la lectura universitaria: más complejidad, mayor atención, más autores, más tiempo (todo el tiempo) y también de un aspecto que la hace más llevadera: es más interesante. También revela una representación que opera en la población de ingresantes: la lectura es una práctica asociada con un área de conocimiento: Lengua. Nos preguntamos si es posible transitar el colegio secundario sin leer en Historia, en Geografía, en Biología. Nuestra experiencia nos indica que también en esos espacios curriculares circulan lecturas, libros de texto, fotocopias, apuntes; la respuesta de los alumnos nos indica no tanto que no hubiera lecturas sino que lo que se leía en Historia, Geografía o Biología no era considerado 'lectura', sino otra cosa, quizá estudio. Una representación que podríamos sintetizar como "Leer no es estudiar, es otra cosa", "Se lee en Lengua, se estudia en Historia o Geografia”. 


\section{La lectura en el nivel superior}

En el tercer eje temático, las representaciones de la lectura en el nivel superior, se recogió, en primer lugar, información de las representaciones sociales de los alumnos en cuanto a lo que consideraban que era leer bien en la universidad. En segundo lugar, para trabajar con la dimensión actitudinal, se hizo un relevamiento de la percepción de los principales obstáculos de lectura y de las conductas que los alumnos asumen frente a estas dificultades.

Acerca de la idea que tienen los estudiantes sobre lo que significa "leer bien en la universidad", la mayoría lo relaciona con la comprensión cabal de los textos, con estar concentrados para lograr identificar las ideas principales del autor o los postulados más importantes de una teoría, también, con llevar al día todas las lecturas de las materias y enriquecer su vocabulario, es decir, apropiarse de los tecnicismos propios de cada campo del saber y poder expresarse correctamente. Encontramos aquí diferente tipo de habilidades: competencias cognitivas (comprensión cabal, atención, concentración, selección de ideas principales), lingüístico-discursivas (vocabulario técnico y expresión) y hábitos de estudio (como llevar al día todas las lecturas).

En este punto, observamos que todas las repuestas se asocian con la finalidad de aprender un contenido específico o con la formación de un lenguaje profesional; también -en coincidencia con el planteo de Carlino (2011)- observamos que, en general, persiste la idea de la lectura como la "extracción de significados dados en un texto", es decir, una práctica pasiva y obediente donde se busca un sentido que ya fue construido por el autor. El desafío es estar atento e identificar aquello que el autor quiso desarrollar, acertar a 'captar' las ideas principales, para eso es necesario mantenerse en forma (llevar al día las lecturas). Aparece la lectura como una tarea que se desarrolla de manera autónoma por el estudiante pero no como un proceso de co-construcción entre los textos, los propósitos, los conocimientos previos del lector y la guía que aportan los docentes. La representación parece suponer que se trata de un proceso de 'absorción', de 'inmersión en los textos' más que de construcción de significados.

Ahora bien, en cuanto a los resultados de las encuestas asociados con los factores que obstaculizan la lectura en la universidad, estos fueron:

-En primer lugar se destaca la complejidad que presentan los textos académicos en cuanto a: "los términos técnicos que utilizan", "la dificultad para encontrar el tema puntual", "los diferentes puntos de vista que plantean." En este caso, observamos que es amplio el número de estudiantes que refieren las dificultades de comprensión que les generan los textos académicos, cabe destacar que quienes lo hacen siempre mencionan problemas que se atribuyen fundamentalmente al texto, especialmente con respecto al vocabulario, el tema y el estilo de escritura. En este caso, no se asume ninguna responsabilidad en el proceso de comprensión que implica un texto, ni se reconocen como propias las dificultades. Esto condiciona las estrategias que puede llegar a seleccionar el alumno para autorregular su lectura, conduciendo en muchas ocasiones a abandonarla sin acertar a poner en práctica alguna que lo ayude a superar los problemas. Si los textos son muy difíciles, no hay mucho que pueda hacer, porque se percibe que la dificultad es externa a sí mismo, está en el texto que se vuelve inabordable.

Otro asunto que se menciona con insistencia es el tiempo de lectura que tienen en relación con la cantidad de material que deben leer para las diferentes materias, lo cual se percibe como insuficiente si se tiene en cuenta que parte de estas lecturas se realizan en horas de clases (en relación con esto, agregamos que la mayoría indicó que dedicaba entre 4 y 6 horas semanales a la lectura de textos de la carrera). 
En menor medida, otros estudiantes apuntaron dificultades físicas o ambientales y económicas para contar con el material: "no suben el material a la plataforma Unpabimodal", "las fotocopias son muy caras", "en las clases no se puede leer, hay mucho bullicio".

Por último, apuntamos los procedimientos y actitudes relacionados con el aprendizaje y la lectura que utilizan los estudiantes ingresantes para enfrentar sus dificultades de lectura. En este punto exponemos los resultados cuantitativos de la pregunta 6: ¿Qué hacés ante las dificultades de lectura que se te presentan?, con varias opciones para responder:

Gráfico 3

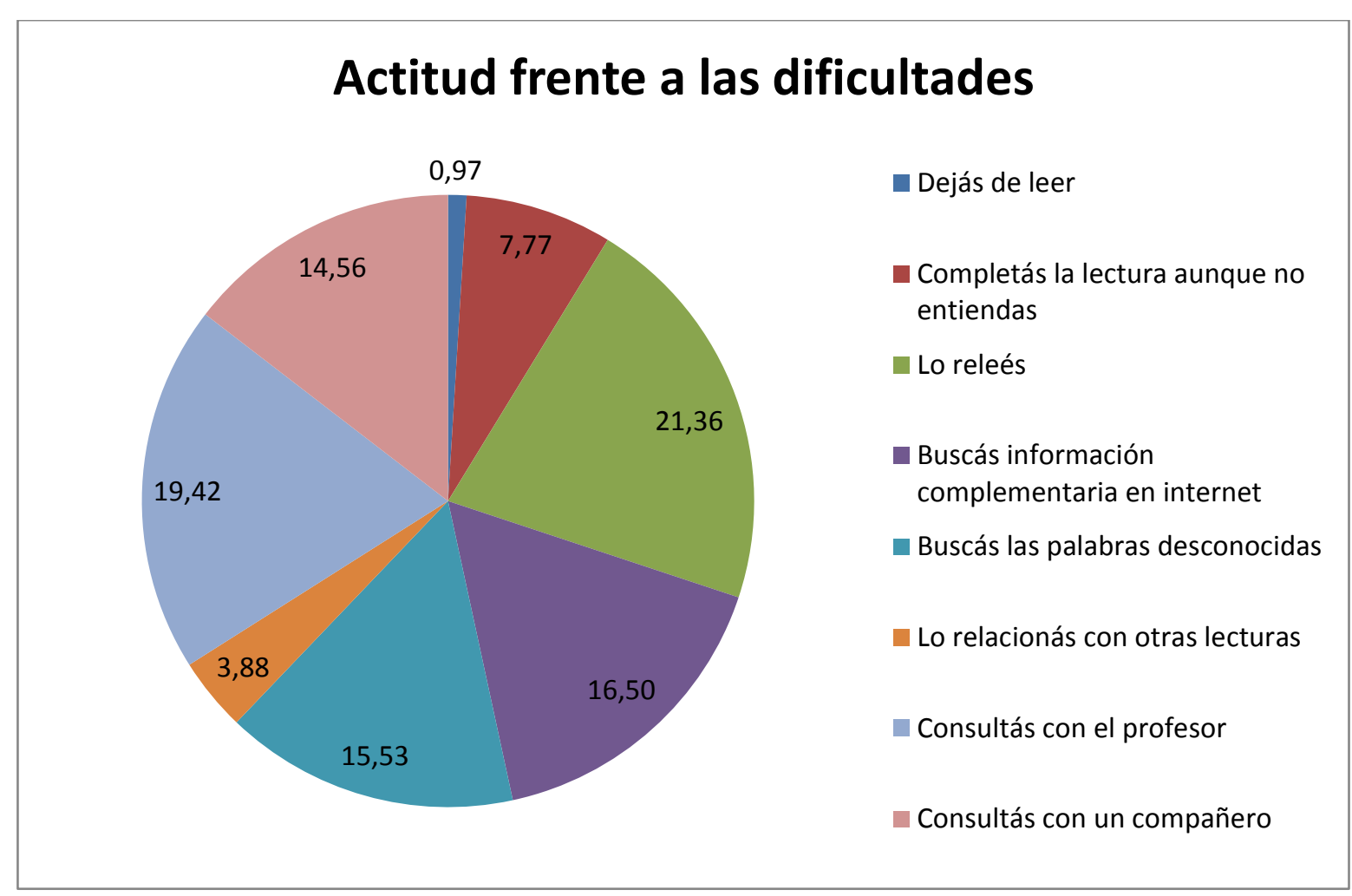

Al respecto, los resultados indican que la relectura $(21,36 \%)$ y la consulta con el profesor $(19,42 \%)$, son las estrategias fundamentales que utilizan los alumnos ingresantes para resolver dificultades de comprensión; en menor medida, buscan información complementaria en Internet sobre el tema $(16,5 \%)$ o consultan diccionarios $(15,53 \%)$. En general, se trata nuevamente de acciones en las que se deposita en otro la tarea de otorgar un sentido al texto o buscan ampliar la información sin considerar las fuentes que consultan. Estos resultados coinciden en parte con los obtenidos hace unos años en una investigación realizada también en la UNPA (Musci, 2015), aunque en la encuesta realizada en aquel momento no aparecía el recurso a Internet. Evidentemente, el perfil de los ingresantes asume nuevos rasgos ${ }^{6}$, la población estudiantil es cambiante y el mundo en el que nos movemos también. El lugar que

\footnotetext{
${ }^{6}$ Por ejemplo, aunque no contamos con estadísticas al respecto, la percepción de los profesores de primer año es que el porcentaje de alumnos ingresantes recién salidos del colegio secundario ha aumentado notablemente en los últimos diez años. Antes, ese grupo social se componía de un porcentaje mayor de "adultos" que retoman trayectos educativos a la vez que tienen obligaciones laborales.
} 
ocupan en las vidas de todos la Web y las herramientas digitales cambian los modos tradicionales de relación con el conocimiento.

En relación con las estrategias con que los alumnos enfrentan el desafío de la lectura, Solé et al (2005) en una investigación acerca de las tareas de lectura y escritura que se proponen y realizan para aprender en el Bachillerato y en la Universidad en España. Estas investigadoras señalan que las tareas más repetidas son subrayar, obtener ideas principales y tomar apuntes, y menos frecuentemente resumir, elaborar síntesis y mapas conceptuales. Concluyen que las tareas favorecedoras del aprendizaje constructivo son escasas y en general conforman un panorama más tradicional de lo esperable. En una encuesta realizada en la Universidad de La Matanza (Bidiña, Luppi, Smael, 2011) los alumnos asignaron utilidad a las siguientes estrategias: realizar tres lecturas, observar los paratextos, realizar una lectura global, leer detenidamente los párrafos, hacer anotaciones marginales, reconocer el plan textual y las marcas de subjetividad.

En síntesis, constatamos que los alumnos utilizan algunas estrategias para trabajar sobre los textos que leen con el propósito de estudiar, pero se trata en general de prácticas más bien pasivas, en las que siguen percibiendo al mundo del texto como ajeno a su propio mundo. Creemos que es necesario extender y profundizar prácticas más activas, sobre todo aquellas que involucran la escritura como herramienta para aprender.

\section{CONCLUSIONES}

Trabajar con las representaciones sociales de la lectura en el primer año de la universidad nos ha permitido construir un perfil lector del alumno ingresante. El análisis de las respuestas de la encuesta realizada nos ha brindado cuestiones de gran importancia para trabajar desde la enseñanza.

En primer lugar, los textos académicos con los que se encuentran los estudiantes universitarios resultan más complejos que los textos escolares con los que estaban acostumbrados a interactuar en el nivel secundario. También se relevan dificultades en torno a las tareas de lectura que se les solicita en el nuevo nivel, creemos que esto sucede porque reproducen las formas de lectura aprendidas en sus experiencias previas.

Por otro lado, los estudiantes utilizan estrategias de lectura y aprendizaje que no implican la construcción de un nuevo conocimiento, sino que apuntan a la reproducción literal de lo que dice el texto o de lo que manifiesta otro actor que detenta poseer el saber.

Por último, y como síntesis de lo anterior, los alumnos ingresantes no se perciben a sí mismos como lectores de textos académicos que puedan autorregular sus propios procesos de comprensión.

Consideramos que contar con esta información es de suma importancia para acortar la brecha entre las representaciones de lectura que poseen los alumnos ingresantes y la que se sostiene desde la comunidad discursiva universitaria. La lectura académica no se aprende de manera espontánea sino que se accede a ella a través de aprendizajes específicos que deben promoverse. El desafío ahora está en visibilizar estas identidades lectoras y propiciar en la universidad prácticas pedagógicas más favorables que incidan positivamente en el desempeño de los estudiantes. 


\section{BIBLIOGRAFÍA}

ABRIC J.-C. (Coord.) (2001). Prácticas sociales y representaciones, México: Ediciones Coyoacán.

ARNOUX E. (2000). "La glotopolítica: transformaciones de un campo disciplinario", en Primer simposio en la maestría en ciencia del lenguaje. SP "Joaquín V. González".

ARAYA S. (2002). Las representaciones sociales: ejes teóricos para su discusión. Cuaderno de Ciencias Sociales 127. Facultad Latinoamericana de Ciencias Sociales. San José de Costa Rica, Costa Rica. Disponible en: http://unpan1.un.org/intradoc/groups/ public/documents/ICAP/UNPAN027076.pdf

BIDIÑA A., LUPPI L. y SMAEL N. (2011). "La lectura en el curso de ingreso. Intervención didáctica. Experiencia piloto en la UNLAM" en Laco, Natale y Ávila, La lectura y la escritura en la formación académica, docente y profesional. Edutecne (e-book).

BOURDIEU, P. y MIZRAJI, M. (1988). Cosas dichas. Buenos Aires: Gedisa.

BOATTO Y., VÉLEZ G. y BONO A. (2016). Aprendizaje a partir de la lectura en el primer año universitario. Transacciones a partir de la lectura. En: La lectura, la escritura y el interés por aprender en la universidad: problemas, saberes y propuestas / Adriana Bono [et al.]; Alicia Vázquez (Coord.).Río Cuarto: UniRío Editora.

CARLINO P. (2011). Leer y escribir en las ciencias sociales en universidades argentinas. Revista Contextos de Educación, Departamento de Cs. de la Educación, Facultad de Cs. Humanas, UNRC. ISSN 1514-2655.

CHARTIER A.-M. y HÉBRARD J. (2002). La lectura de un siglo a otro. Discursos sobre la lectura (1980-2000). Barcelona, Gedisa.

COIRO J. (2003). Comprensión de la lectura en Internet: ampliando lo que entendemos por comprensión de la lectura para incluir las nuevas competencias. En EDUTEKA, jul. 2003. Disponible en: http://www.eduteka.org/ediciones/recomendado17-8a.htm

DI STÉFANO M. y PEREIRA C. (1997). Representaciones sociales en el proceso de la lectura. Signo y Seña 8. Pp.371-338.

DI STÉFANO M. y PEREIRA C. (2004). La enseñanza de la lectura y la escritura en el nivel superior: procesos, prácticas y representaciones sociales. Textos en contexto 6. Pp. 2437.

DI STÉFANO M. y PEREIRA C. (2014). Aplicación de una encuesta sobre recorridos y prácticas lectoras en alumnos del CBC (Ciclo Básico Común) de la Universidad de Buenos Aires. Avance de resultados. Enunciación 19, (1). pp. 81-92

FERNÁNDEZ A. O. J. y OJEDA RAMÍREZ M. M. (2018). La lectura no utilitaria en la universidad en la era digital. Un análisis multivariante que ubica el texto impreso en la lectura de literatura. Palabra Clave 7 (2).

GOODMAN K. (1982). El proceso de lectura: consideraciones a través de las lenguas y del desarrollo en Ferreiro, E. y Gómez Palacio, M. (Comp.) Nuevas perspectivas sobre los procesos de lectura y escritura, pp. 13-28.

GUTIÉRREZ VALENCIA A. (2009). El estudio de las prácticas y las representaciones sociales de la lectura: génesis y el estado del arte. Anales de Documentación (12), pp. 53-67. Universidad de Murcia Espinardo, España.

JODELET D. (1986). La representación social: fenómenos, concepto y teoría. En Moscovici, S. (Ed) Psicología Social II: Pensamiento y vida social (pp.469-493). Barcelona: Paidós.

JODELET D. (1989). Representaciones sociales: un campo en expansión. En AA.VV., Les représentations sociales (pp. 31-61). París: P. U. F.

LAHIRE B. (2004). Sociología de la lectura. Barcelona: Gedisa. 
MOSCOVICI S. (1989). Des représentations collectives aux représentations sociales: éléments pour une histoire. Les représentations sociales, 5, 79-103.

MUSCI M. (2015). Percepciones de alumnos universitarios sobre sus propias prácticas de lectura y escritura: géneros, propósitos, estrategias y dificultades. En: Gabbiani y Orlando, Escritura, lectura y argumentación en contextos educativos en el Río de la Plata. UdelaR. CSIC. Montevideo. SBN 978-9974-0-1246-2.

NOGUEIRA S. y ROBBIO M. (2010). Leer y escribir como universitario. En: Nogueira, S. (coord.) Estrategias de lectura y escritura académica; estudio y ejercitación de la citación, la textualidad, la explicación y la argumentación (pp.9-43). Buenos Aires: Biblos.

ORTIZ C. (2013). Las representaciones sociales: un marco teórico apropiado para abordar la investigación social educativa. Revista en Ciencias XIX (enero-marzo. 2013), pp. 183193. Universidad de Zulia, Maracaibo, Venezuela.

PARODI G. (2011). Comprensión de textos escritos. Buenos Aires: Eudeba.

RAMIREZ LEYVA E. (Coord.)(2015). Tendencias de la lectura en la universidad. México: UNAM, Instituto de Investigaciones Bibliotecológicas y de la Información. Disponible en: http://universidadeslectoras.org/dt/finder/tendencias_lectura_universidad.pdf

SOLÉ I. (2000). Estrategias de Lectura. Barcelona: Graó.

SOLÉ I., MATEOS M., MIRAS M., MARTÍN E., CASTELLS N., CUEVAS I., GRÀCIA M. (2005). Lectura, escritura y adquisición de conocimientos en Educación Secundaria y Educación Universitaria. En Revista Infancia y Aprendizaje, vol. 28, № 3: pp. 329 347.

SOSA DE MONTYN S., CONTI DE LONDERO M., FURLAN M., LOSS T., MAZZUCHINO M. (2006). ¿Por qué es necesario saber escribir bien en la universidad? Representaciones sociales de escritura de alumnos de la Facultad de Lenguas de la Universidad Nacional de Córdoba. Presentado en Publicación Digital del Primer Congreso de Lectura y Escritura del Litoral. Universidad Nacional del Litoral. Santa Fe. Disponible en: http://www.fhuc.unledu.ar/escrituraylectura. 


\section{ANEXO}

\section{Encuesta sobre representaciones sociales de la lectura}

A través de la presente encuesta intentamos obtener información sobre representaciones sociales de la lectura en nuestra universidad. El cuestionario es anónimo. No hay respuestas buenas o malas. Lo que nos interesa son respuestas verdaderas. Muchas gracias por tu colaboración.

Proyecto "Regulaciones discursivas: intervenciones glotopolíticas en la Universidad"

\section{Algunos datos personales:}

Sexo: $\quad$ Edad: $\quad$ Ingresante/ Recusante/ o año de la carrera que cursa:

\section{P.1. Área o carrera a la que perteneces:}

Trabajo social Turismo Geografía Psicopedagogía Comunicación social Otra:

P.2. ¿Cuál de las siguientes frases explica mejor tu relación con la lectura? (Escogé sólo una respuesta)
a) No puedo vivir sin la lectura
d) Me gusta poco leer
b) Me gusta mucho leer
e) No me gusta nada leer
c) Me gusta leer de vez en cuando

P.3. ¿Cuáles son las razones principales que te llevan a leer? (Podés escoger tres opciones, indicalas en orden de importancia: $1,2,3$ )
a) Informarme sobre algún tema de interés
f) Crecimiento personal
b) El deseo de conocer cosas nuevas
g) Para ser un mejor profesional
c) Cumplir con las actividades académicas
h) Para huir de las preocupaciones
d) Deseo expresarme mejor
i) Para ser culto
e) El placer que encuentro en la lectura
j) Otra, ¿cuál?

P.4. Pensá en las prácticas de lectura que realizabas en la escuela secundaria, ¿qué diferencias encontrás con las que realizás ahora en la universidad?

\section{P. 5. ¿Por qué es importante leer bien en la universidad?}


P.6. ¿Con qué propósitos asociás la lectura en la universidad? (Escogá tres opciones e indicá en orden de importancia: $1,2,3$ )

a) Aprender los contenidos de una materia

b) Responder a preguntas planteadas por el o la docente

c) Identificar los postulados más importantes que representan a un autor o una teoría

d) Diseñar un proyecto

e) Realizar una exposición oral

f) Participar en discusiones grupales

g) Asistir a eventos académicos

h) Escribir artículos o ponencias

i) Trabajar en el marco de un proyecto de investigación

j) Responder a una evaluación escrita

k) Elaborar un escrito académico. ¿De qué tipo?

1) Otros ¿Cuál?

P. 7. ¿Cuáles son los tipos de texto que leés con más frecuencia en la carrera? (podés elegir varias opciones)
a) Materiales elaborados por el profesor
g) Informes de investigación
(guías, notas de clase)
h) Libros de consulta general (enciclopedias
b) Apuntes de clase propios
y diccionarios)
c) Apuntes de clase de otro (s) compañero (s)
i) Libros o capítulos propios de la carrera
d) Resúmenes de libros o de artículos
j) Literatura (novelas, cuentos, poesía)
e) Artículos científicos
k) Páginas web, blogs
f) Documentos periodísticos (noticias,
l) Otro ¿cuál?

crónicas, artículos de opinión, editoriales)

P. 7. ¿Cuántas horas semanales le dedicás a la lectura de textos de tu carrera?

Hasta 3 horas semanales

Más de 6 horas semanales, ¿cuánto?

De 4 a 6 horas semanales

P.8. ¿Qué tipo de soporte preferís para leer documentos de la carrera?

Digital Impreso Ambos

P.9. Si leés material digitalizado, ¿a través de qué dispositivo accedés a él?

Tablet

Celular

Computadora
Kindle/Kobo

Otro, ¿cuál?

\section{P.10. ¿Creés que el soporte dificulta o facilita lectura? ¿Por qué?}


P. 11. ¿Cuáles creés que son los obstáculos de lectura más frecuentes en la universidad? ¿Podés dar un ejemplo?

P.12. ¿Qué hacés ante las dificultades de lectura que se te presentan?

Dejás de leer

Completás la lectura aunque no entiendas el texto

Buscás información complementaria en Internet

Buscás en un diccionario las palabras desconocidas

Releés

Consultás con el profesor

Consultás con un compañero

Otras estrategias: 